\title{
Sirtinol regulates the balance of Th17/ Treg to prevent allograft rejection
}

\author{
Qing Ye ${ }^{1 \dagger}$, Mingjian Zhang ${ }^{2 \dagger}$, Yang Wang ${ }^{3 \dagger}$, Shangxi Fu' ${ }^{1}$, Shu Han ${ }^{1}$, Liming Wang ${ }^{1 *}$ and Quanxing Wang ${ }^{2^{*}}$
}

\begin{abstract}
Background: Current immunosuppressive medications used after transplantation induce significant toxicity, and a new medication regimen is needed. Based on recent research, Sirt1 exerts a proinflammatory effect on the immune response. Sirtinol is a Sirt1 inhibitor, but its impact on allograft rejection and its molecular mechanisms of action have not yet been reported.

Resluts: In this study, we examined the effect of sirtinol on prolonging allograft survival in a mouse cervical heterotopic heart transplantation model. Based on an examination of the allograft, allografts from sirtinol-treated recipients show significantly lower levels of IL-17A expression and higher levels of Foxp3 expression. In vivo, sirtinol reduces the proportion of Th17 cells and increases the proportion of Treg cells in splenocytes from recipients. In vitro, sirtinol reduces the proportion of Th17 cells and decreases the expression of IL-17A and RORYt in an isolated CD4 ${ }^{+} \mathrm{T}$ cell population. Moreover, we identified synergistic effects of sirtinol and FK506 on prolonging allograft survival, and sirtinol synergizes with FK506 to promote Foxp3 expression.
\end{abstract}

Conclusion: Sirtinol, a Sirt1 inhibitor, may be a promising immunosuppressive drug to prevent the rejection reaction in combination with FK506.

Keywords: Sirtinol, Allograft rejection, Th17/Treg

\section{Background}

Organ transplantation is the final therapeutic schedule for most patients with end-stage organ disease. According to numerous studies, calcineurin inhibitors (CNIs) significantly improve short-term solid organ graft survival in transplant recipients. The rate of acute rejection has been dramatically reduced and the 1-year survival rate has clearly increased since CNIs have been administered to patients after transplantation. However, the long-term used of CNIs might result in various sideeffects, such as renal toxicity, neurotoxicity and increased risks of infection and cancer [1]. CNIs are now thought

\footnotetext{
*Correspondence: wanglimingsmmu@163.com;

wangqx@immunol.org

${ }^{\dagger}$ Qing Ye, Minjian Zhang and Yang Wang contributed equally to this work

${ }^{1}$ Institute of Organ Transplantation, Changzheng Hospital, Second

Military Medical University, Shanghai, China

${ }^{2}$ National Key Laboratory of Medical Immunology, Second Military

Medical University, Shanghai, China

Full list of author information is available at the end of the article
}

to be necessary for preventing allograft rejection, but reduced doses are preferred [2].

Based on traditional transplantation immunology, APCs from donors or recipients activate $\mathrm{T}$ cells through direct recognition and indirect recognition, respectively. Next, T helper cells release interferon (IFN)- $\gamma$, interleukin (IL)-2, tumor necrosis factor (TNF) and other types of cytokines to mediate rejection [3, 4]. Therefore, CNIs are administered with FKBP12 to inhibit the activation of nuclear factor of activated T-cells (NF-AT) and the subsequent transcription of the IL-2 gene to prevent the reject reaction.

The Th17/Treg balance was strongly associated with allograft rejection in some recent studies [5, 6]. Th17 cells are important for mediating acute and chronic rejection, whereas Tregs contribute to the induction and maintenance of tolerance of the allografts in recipients $[7,8]$. However, CNIs do not effectively restrain the rejection reaction mediated by Th17 cells, and CNIs have been reported to cause a Th17/Treg imbalance [9]. Therefore, therapeutic strategies aimed at manipulating the Th17/ 
Treg balance are now considered the most promising approach for preventing allograft rejection.

In recent years, immunologists have paid more attention to epigenetic regulation, which is thought to be the precise regulatory mechanism in immune cells. Transplant scientists focus on the mechanism by which epigenetics regulate rejection, particularly by preventing chronic rejection. Sirtuins are NAD-dependent class III histone deacetylases (HDACs) that play critical roles in diverse physiological processes, such as prolonging the life-span, metabolism, cell senescence, cell autophagy/ apoptosis, autoimmunity, oxidative stress, and inflammation [10]. Sirtuins include seven members known as Sirtuin1-Sirtuin7. Among them, Sirtuin1 (Sirt1) specifically acts as an epigenetic regulator that modulates the activity of several transcription factors important for immune function.

Sirt1 was initially suggested to exert a primarily anti-inflammatory function [11-13]. However, recent research focusing on $\mathrm{T}$ cells has shown that Sirt1 exerts a proinflammatory effect on the immune response. On one hand, Sirt1 was identified as a negative regulator of Treg cell function by deacetylating Foxp3, the signature transcription factor of Treg cells $[14,15]$. On the other hand, Sirt1 might positively regulate the function of Th17 cells by modulating RORyt activity [16]. Based on the findings from these studies, Sirt1 may be a new potential therapeutic target to promote immunosuppression in transplant recipients.

As a Sirt1 inhibitor, sirtinol has displayed anti-tumor [17-19] and anti-inflammatory properties [20, 21], but its impact on allograft rejection and its molecular mechanisms of action have not yet been reported. In the present study, we established a mouse model of cervical heterotopic heart transplantation and evaluated the impact of sirtinol on cardiac allograft rejection. Notably, sirtinol synergizes with tacrolimus to prevent mouse cardiac allograft rejection, as sirtinol inhibits the Th17-mediated rejection and increases the proportion of Treg cells.

\section{Methods}

\section{Mice and reagents}

Mice were obtained from the Second Military Medical University Animal Supply Center and housed in a specific pathogen-free facility. C57BL/6 (aged 10-14 weeks, male) mice were used as recipients and $B A L B / C$ mice (aged 8-12 weeks, male) were used as heart donors. All animal experiments were conducted under the experimental protocol approved by the Ethics Review Committee for Animal Experimentation of the Second Military Medical University. We purchased sirtinol and tacrolimus from Selleck Chemicals (Boston, MA, USA). Sirtinol and tacrolimus were dissolved in dimethyl sulfoxide (DMSO), and DMSO was used as a control.

\section{Heart transplantation model}

The cervical heterotopic heart transplantation mouse model was prepared as previously described [22]. Recipient mice were intraperitoneally injected with sirtinol $(1 \mathrm{mg} / \mathrm{kg} /$ day $)$, FK506 (1 mg/kg/day), or both sirtinol $(1 \mathrm{mg} / \mathrm{kg} /$ day $)$ and FK506 $(1 \mathrm{mg} / \mathrm{kg} /$ day $)$. The study point being evaluated was defined as complete cessation of the heartbeat. Survival rates of cardiac grafts were monitored by palpation.

\section{Histology examination}

Cardiac allografts were excised at 7 or 12 days post-transplantation, and fixed with 5\% neutral paraformaldehyde, routinely processed, and embedded in paraffin. Histological sections for light microscopy were cut to a thickness of $4 \mu \mathrm{m}$ and stained with hematoxylin and eosin. Infiltrating cells were counted under at high magnification $(\times 40)$ using ImagePro Plus software.

RNA isolation, reverse transcription, and quantitative PCR Total RNA was extracted from allografts using TRIzol reagent (Invitrogen, Carlsbad, CA, USA), according to the manufacturer's instructions; cDNAs were synthesized using an oligo $\mathrm{d}(\mathrm{T})$ primer (Applied Biosystems, Foster City, California, U.S.) and a Superscript III Reverse Transcriptase Kit (Invitrogen). A StepOne ${ }^{\mathrm{TM}}$ Real-Time PCR System (Applied Biosystems) and a SYBR RT-PCR kit (Takara, Japan) were used for the quantitative realtime PCR analysis. All reactions were conducted in a $20 \mu \mathrm{l}$ reaction volume in triplicate. The relative expression levels of a target gene were normalized to GAPDH expression. The specificity of qPCR was verified with a melting curve analysis and agarose gel electrophoresis. Primer sequences used in the RT-PCR analysis are shown in Table 1 [23].

\section{T cells isolation and flow cytometry}

We isolated $\mathrm{T}$ lymphocytes from spleens. After preparing a single cell suspension and counting the cells, we used magnetic beads (Miltenyi Biotec) to isolate $\mathrm{CD}^{+}$cells. Isolation was performed in the dark, and the total number of cells added to the LS column was restricted to $1 \times 10^{8}$. Anti-CD4, anti-CD25, anti-IFN- $\gamma$, anti-IL-4, anti-IL-17A, and anti-Foxp3 antibodies were purchased from BioLegend. Intracellular straining for IFN- $\gamma$, IL-4, IL-17A and Foxp3 was performed using the Prem/Fix Buffer set, according to the manufacturer's instructions [24]. After staining, cells were suspended in PBS $+1 \%$ FBS and analyzed using an Accuri C6 flow 
Table 1 RT-PCR primer sequence

\begin{tabular}{ll}
\hline Primer & Sequence \\
\hline RORyt & Forward 5'-TCA CCT GAC CTA CCC GAG G-3' \\
& Reverse 5'-TCC AAG AGT AAG TTG GCC GTC-3' \\
GAPDH & Forward 5'-AGG TCG GTG TGA ACG GAT TTG-3' \\
& Reverse 5'-TGT AGA CCA TGT AGT TGA GGT CA-3' \\
IL-17A & Forward 5'-TCG CCA TTC AGC AAG AAA TCC-3' \\
& Reverse 5'-CAC AGG TGC AGC CAA CTT TTA-3' \\
IFN- $y$ & Forward 5'-GAA CTG GCA AAA GGA TGG TGA-3' \\
& Reverse 5'-TGT GGG TTG TTG ACC TCA AAC-3' \\
IL-4 & Forward 5'-GGT CTC AAC CCC CAG CTA GT-3' \\
& Reverse 5'-GCC GAT GAT CTC TCT CAA GTG AT-3' \\
FOXP3 & Forward 5'-TCA AGT ACC ACA ATA TGC GAC C-3' \\
& Reverse 5'-CCA TCG GAT AAG GGT GGC A-3' \\
\hline
\end{tabular}

cytometer from BD Biosciences (San Jose, CA, USA). We also detected the serum IFN- $\gamma$, IL-4, and IL-17 levels using enzyme-linked immunosorbent assay (ELISA) kits, according to the manufacturer's protocol (R\&D Systems, Minneapolis, MN, USA).

\section{Statistical analysis}

Data were analyzed with GraphPad Prism 5.0 software (GraphPad, Inc., La Jolla, CA, USA). All normally distributed data were displayed as means \pm standard deviations (SD). Measurements between two groups were analyzed using Student's $t$ test or the Mann-Whitney U test. Allograft survival was assessed using a log-rank (Mantel-Cox) test. A P value $<0.05$ was considered significant.

\section{Results}

\section{Sirtinol prolongs the survival of cardiac allografts}

We developed a fully MHC-mismatched murine cervical heterotopic cardiac transplant model in which C57BL/6 mice were transplanted with the hearts from BALB/C mice and randomized to treatment with sirtinol $(1 \mathrm{mg} /$ $\mathrm{kg} /$ day i.p. after transplantation) or DMSO as a vehicle control to assess the effect of sirtinol on immune responses. The median survival time of the DMSO group was 7 days, but the administration of sirtinol prolonged the median survival of the allograft to 10 days (Fig. 1a).

Next, five allografts from each experimental group were harvested on day 7 post-transplantation and subjected to histological analysis. The histological analysis clearly revealed a rejection reaction in the DMSO group, whereas the sirtinol group was characterized by less infiltration (Fig. 1b, c).

Based on these results, sirtinol prolongs the survival of MHC fully mismatched cardiac allografts, but the exact mechanism remains unknown.

\section{Sirtinol regulates the Th17/Treg balance}

Next, we examined the impact of sirtinol on the expression of inflam-matory cytokines and regulatory molecules. Allografts from the DMSO and sirtinol groups were harvested on day 7 after transplantation for quantitative PCR analysis. T cells play a primary role in the acute rejection reaction, and thus we first evaluated the expression of IFN- $\gamma$, IL-4, IL-17A and Foxp3. Compared with allografts derived from the DMSO group, allografts from sirtinol-treated recipients showed significantly lower levels of IL-17A and higher Foxp3 expression. In contrast, the expression of IFN- $\gamma$ and IL- 4 was not significantly different between the DMSO group and sirtinol group (Fig. 2a). The immunofluorescence analysis also revealed the same results (Fig. 2b).

Given the role of Foxp3 in Treg cells, we evaluated the number of Treg cells in hosts from the DMSO and sirtinol groups. We isolated cells from spleen at 7 days after transplantation and then analyzed cells using flow cytometry. Indeed, the sirtinol treatment significantly increased the proportion of Treg cells among $\mathrm{CD} 4^{+} \mathrm{T}$ cells in the spleen (Fig. 3b). The effects of sirtinol on cytokine production, including IL-4, IFN- $\gamma$ and IL-17A, were also assessed using intracellular staining. A significant decrease in IL-17A staining was observed in the sirtinol group (Fig. 3b). No obvious changes in IL- 4 and IFN- $\gamma$ expression were observed between the DMSO group and sirtinol group. We also detected the serum IFN- $\gamma$, IL-4, and IL-17A levels using ELISA kits and obtained the same results (Fig. 3c).

Sirtinol suppressed IL-17 expression in allografts; thus, we examined the impact of sirtinol on the differentiation of Th17 cells. Naive CD4 $4^{+} \mathrm{T}$ cells were isolated and then polarized to the Th17 lineage in the presence of sirtinol or DMSO, as previously described [25]. According to the results of the flow cytometry analysis of polarized T cells, sirtinol significantly suppressed the production of Th17 cells (Fig. 4a), and the western blot analysis indicated that sirtinol reduced the expression of RORyt and promoted Foxp3 expression (Fig. 4b). The qPCR analysis further confirmed that sirtinol down-regulated IL-17A and RORyt expression, but up-regulated Foxp3 expression (Fig. 4c). Based on these results, sirtinol may regulate the balance between Th17 and Treg cells to prolong cardiac allograft survival.

\section{Sirtinol functions synergistically with CNIs to alleviate cardiac allograft rejection}

Since sirtinol and CNIs use different mechanisms to restrain rejection, we treated mice with sirtinol and tacrolimus after transplantation. Co-administration of sirtinol and tacrolimus prolonged the median allograft survival to 21 days, whereas the administration of 


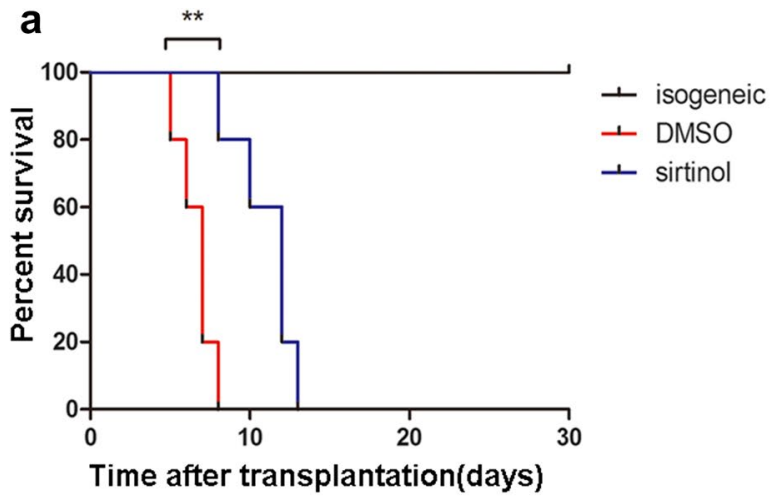

b

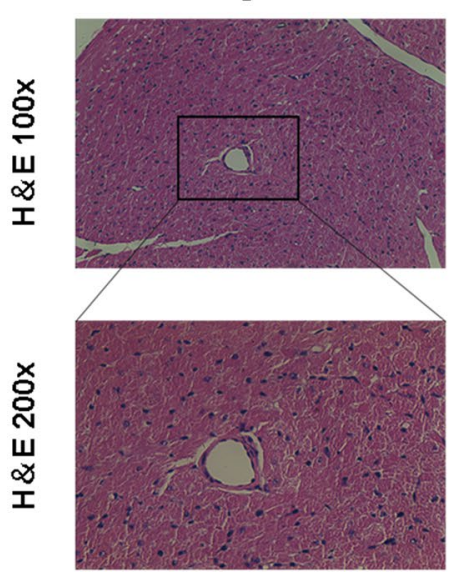

sirtinol
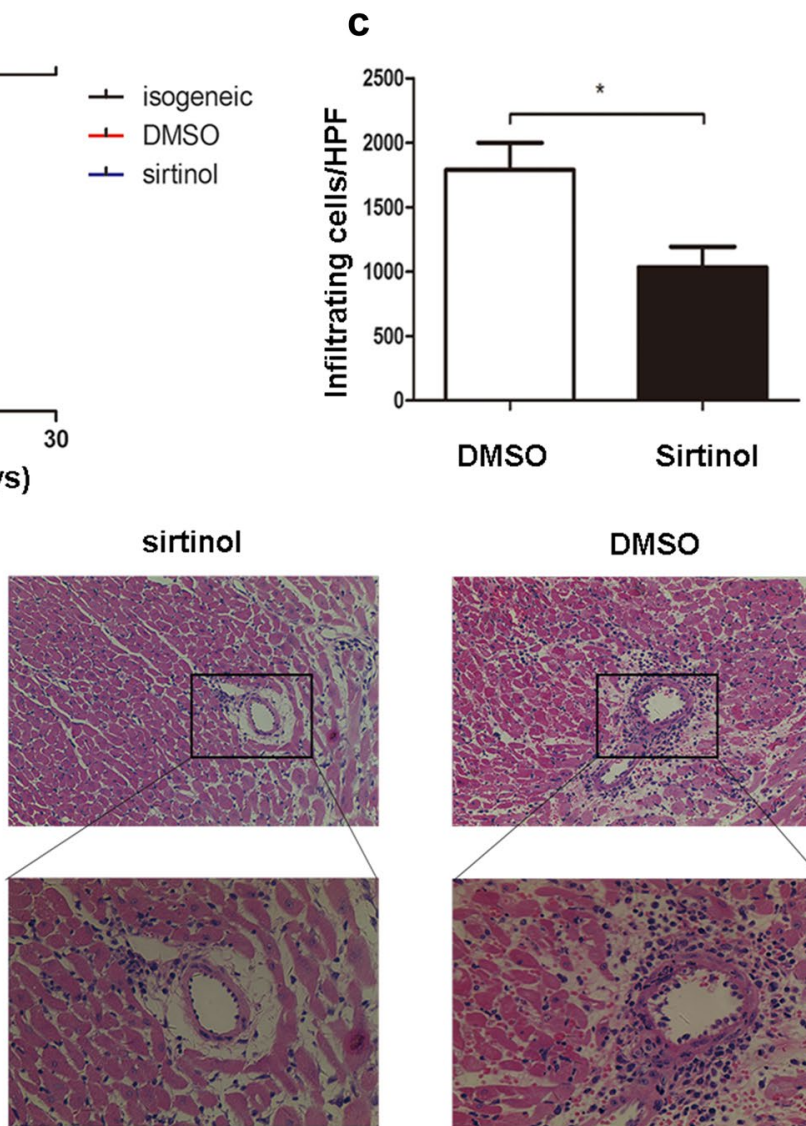

DMSO

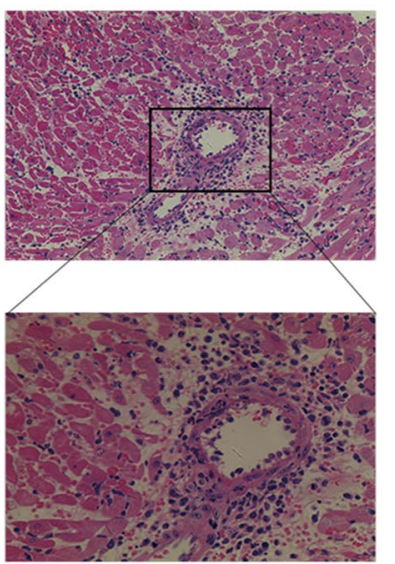

Fig. 1 Sirtinol prolongs the cardiac allograft survival in murine cervical heterotopic cardiac transplant model. a Fully MHC-mismatched cardiac allograft recipients (BALB/C to C57BL/6) were treated with DMSO, sirtinol (1 mg/kg/day) by intraperitoneal injection since the day receiving transplantation until when cardiac arrest occurred. Graft survival was assessed every day. b Pathological examination of allografts harvested on day 7 post-transplantation. Shown are section graphs from the low power field and high power field. c Infiltrating cells per high field were counted from five mice per group. ${ }^{*} \mathrm{P}<0.05,{ }^{* *} \mathrm{P}<0.01$, compared with DMSO group

tacrolimus prolonged the median allograft survival to 16 days (Fig. 5a).

Next, five allografts from each group were harvested on day 12 post-transplantation and subjected to a histological analysis. Compared with that in the tacrolimus group, co-administration of sirtinol and tacrolimus completely prevented inflammatory infiltration, but did not change myocardial structure (Fig. 5b, c).

We conducted a qPCR analysis to analyze the grafts and confirm these results. Compared with allografts derived from the tacrolimus-treated groups, allografts from the tacrolimus/sirtinol group showed significantly lower levels of IL-17 expression. No significant difference in IL-17 expression was observed between the sirtinol and tacrolimus/sirtinol groups, indicating that sirtinol alone reduced IL-17 expression (Fig. 5d). Interestingly, a synergistic effect of sirtinol and FK506 on Foxp3 expression was detected (Fig. 5e). Based on these data, sirtinol functions synergistically with tacrolimus to promote the survival of cardiac allografts.

\section{Discussion}

Lifelong immunosuppression can result in severe side effects, such as cancer, infection, diabetes, cardiovascular diseases, etc. Currently used immunosuppressive medications cause organ toxicity and do not effectively prevent chronic rejection, limiting long-term allograft survival [26]. Therefore, a new therapeutic target with minimal side effects and adequate immunosuppression is required. In this study, the class III HDAC Sirt1 played a critical role in the $\mathrm{T}$-cell-mediated immune response after organ transplantation, and the inhibition of Sirt1 by sirtinol protected the allograft from inflammatory cell infiltration and prolonged graft survival.

The immunosuppressive effects of conventional $\mathrm{Zn}$ dependent class I and II HDAC inhibitors are widely 

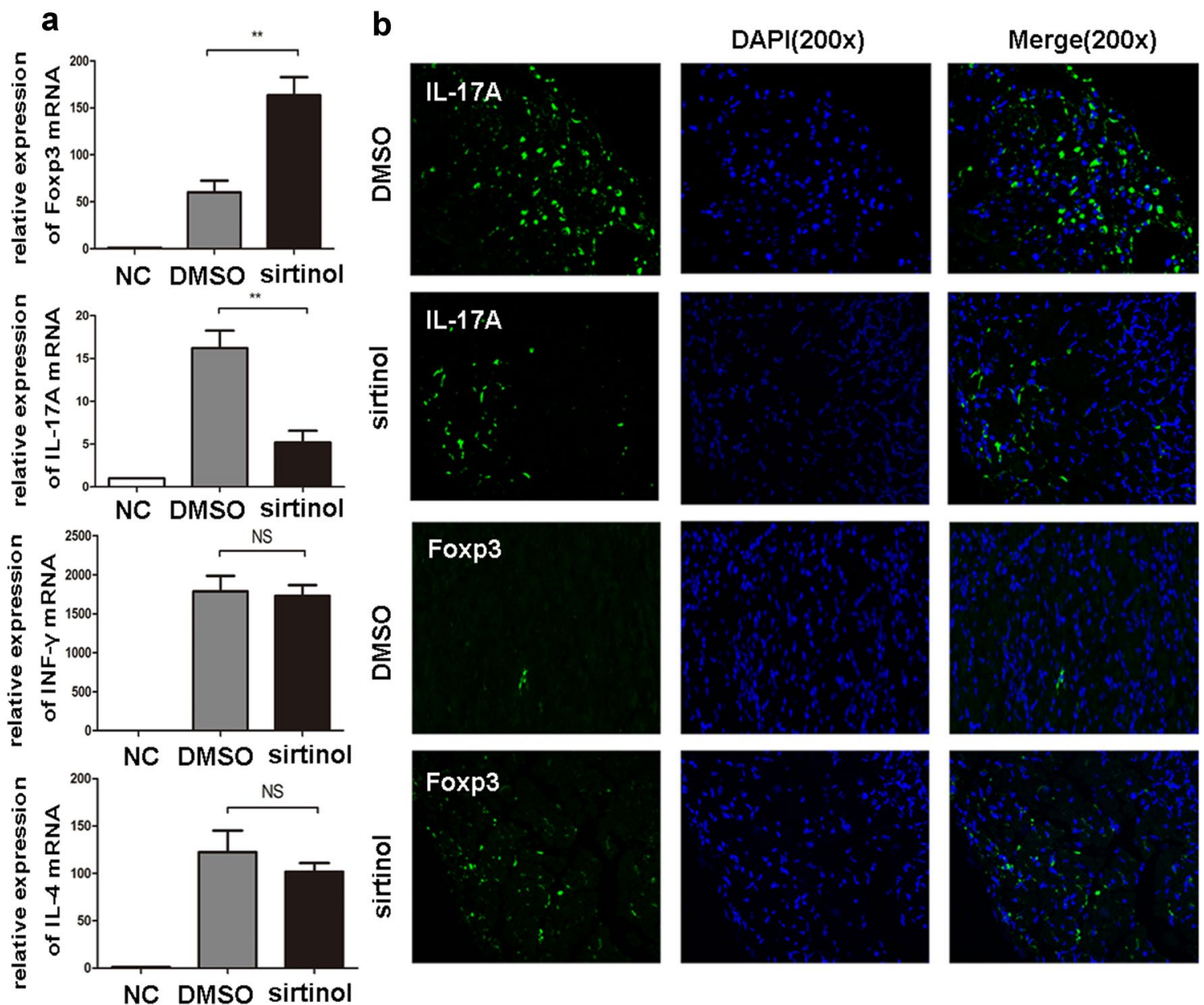

Fig. 2 The effect of sirtinol on the expressions of inflammatory cytokines. a The allografts were harvested 7 days after transplantation. IL-17A, Foxp3, IFN- $y$ and IL-4 mRNA were measured by qPCR. The data are expressed as mean $\pm S D(n=5)$. ${ }^{*} P<0.05,{ }^{* *} \mathrm{P}<0.01$, compared with DMSO group. NC, negative control represented cardiac graft from isotransplantation. b Immunofluorescence examination of allografts harvested on day 7 post-transplantation. Shown are section graphs from high power field

accepted. The class III HDACs were discovered later than the other two classes, and the role of class III HDACs in immune responses remains controversial. In this study, the Sirt1 inhibitor sirtinol clearly prolonged cardiac allograft survival. Based on the GPCR and flow cytometry results, sirtinol may affect Treg and Th17 cells to limit immune responses.

According to an increasing number of recent studies, Th17 cells play a key role in T-cell-mediated acute rejection. An increased level of IL-17A was observed in the immune rejection reactions in several organs, including the lung and kidney. IL-17A inhibition clearly prolongs allograft survival [27]. In addition, Th17 cells may also participate in chronic rejection. As shown in the study by Yuan et al. Th17 cells mediate chronic inflammation and vasculopathy in the model of cardiac allograft vasculopathy in T-bet ${ }^{-1-}$ recipients [28]. In animals, Th17 cells promote allograft fibrosis by inducing chronic inflammation $[29,30]$. Th17 cells secrete IL-17A and IL-21 to mediate chronic rejection [31].

Treg cells, an important $\mathrm{T}$ cell subset, also regulate acute rejection post-transplantation. Studies have shown a positive correlation between the population of Treg cells and allograft survival in tissues such as the lung, kidney and liver [32]. In hematopoietic stem cell transplantation, acute or chronic graft-versus-host disease (GVHD) occurs in the mesentery because of low Treg cell counts [33]. Based on research presented above, we consider that the Sirt1 inhibitor sirtinol, which regulates the Th17/Treg balance, has outstanding potential as an immunosuppressive medicine.

Among the immunosuppressive medicines, CNIs are the cornerstone of immunosuppressive therapy posttransplantation and are the main cause of side effects. 

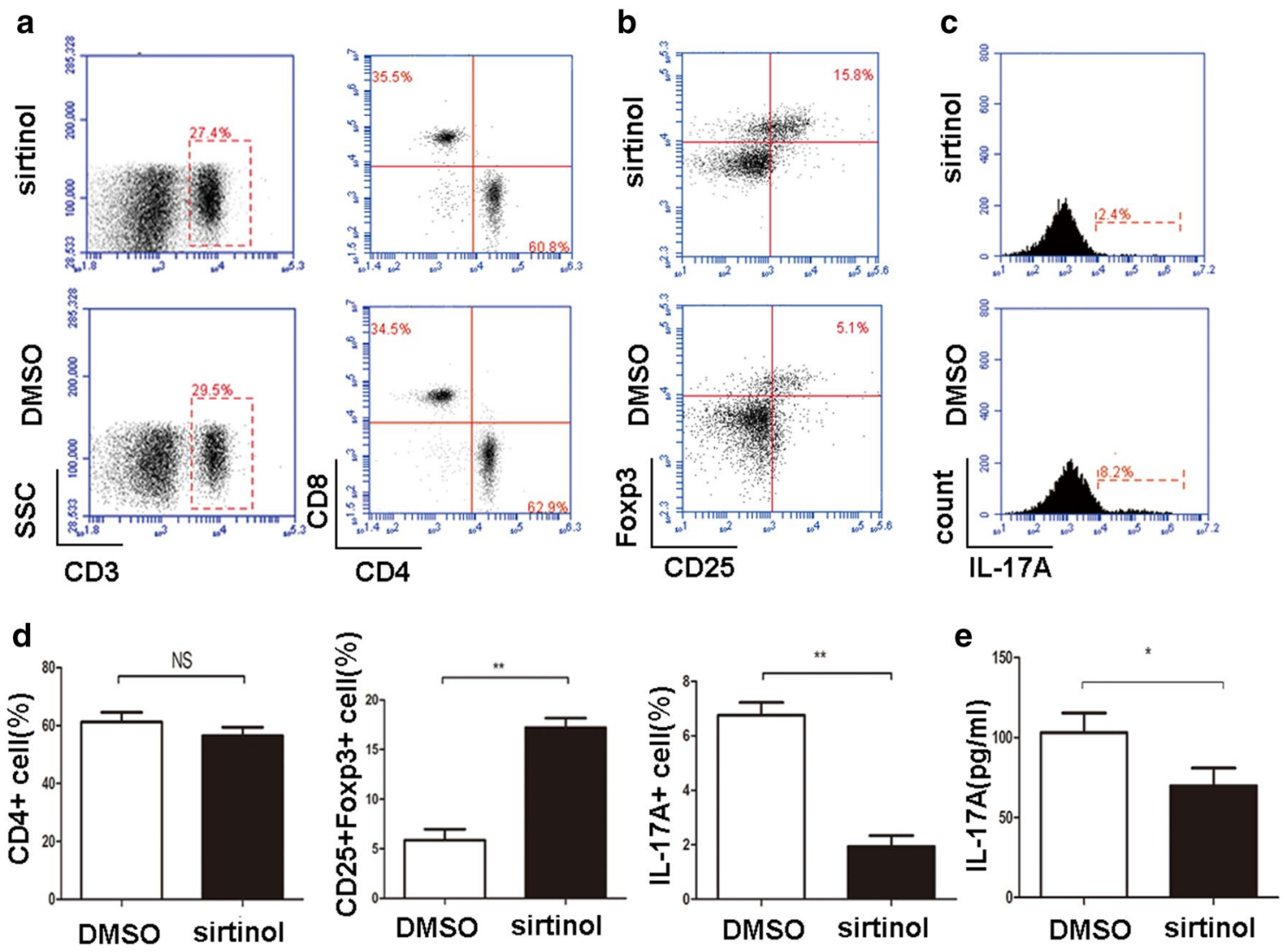

Fig. 3 The effect of sirtinol on the proliferation and differentiation of T cells subset. $\mathbf{a}$ The recipient spleens were harvested 7 days after transplantation. $\mathrm{CD}^{+} \mathrm{T}$ cells, $\mathrm{CD} 4^{+} \mathrm{T}$ cells and $\mathrm{CD} 8^{+} \mathrm{T}$ cells from splenocyte were measured by flow cytometry. $\mathbf{b}$ The recipient spleens were harvested 7 days after transplantation. $\mathrm{CD} 4^{+} \mathrm{T}$ cells were isolated and $\mathrm{CD} 25^{+}$and Foxp $3^{+} \mathrm{T}$ cells were measured by flow cytometry. Shown are representative of five separate experiments. c The recipient spleens were harvested 7 days after transplantation. CD4 ${ }^{+} \mathrm{T}$ cells were isolated and IL-17A ${ }^{+} \mathrm{T}$ cells were measured by flow cytometry. Shown are representative of five experiments. $\mathbf{d}$ Percentages of $\mathrm{CD}^{+}, \mathrm{CD}_{2} 5^{+} \mathrm{Foxp} 3^{+}$and IL-17A $\mathrm{A}^{+}$cells were presented. e ELISA assays were performed to test the IL-17A levels on day 7 after transplantation. The data are expressed as mean $\pm S D(n=5)$. ${ }^{*} P<0.05$, **P $<0.01$, compared with DMSO group

They inhibit IL-2 synthesis to prevent the activation and proliferation of $\mathrm{T}$ lymphocytes. However, the effect of inhibiting Th17 cells is not achieved because of the different activation mechanism of Th17 cells. Thus, we proposed the hypothesis that clinicians should choose a mild immunosuppressant to inhibit Th17 cell-mediated rejection under conditions of long-term CNI use to prolong graft survival. Finally, our research results verify this hypothesis, as the combination of sirtinol and FK506 is more effective at preventing allograft rejection than either of drug alone. Thus, the administration of sirtinol along with a low dose of FK506 may achieve a satisfying immunosuppressive effect with fewer side effects.

FK506, a second generation CNI, inhibits calcineurin activity by a unique, small molecule-mediated, protein-protein interaction. FK506 binds to FKBP12, and this binary complex binds to calcineurin and inhibits its phosphatase activity and downstream signaling. Then, the IL-2 synthesis is inhibited and Th1 cells are not activated because of the lack of IL-2. According to the results from our present study, sirtinol suppresses the production of Th17 cells; therefore, the combination of FK506 and sirtinol should suppress Th cell production more comprehensively than either drug alone. This hypothesis is consistent with our observations in the current study. In addition, CsA and FK506 use different intracellular receptors, but they inhibit cell signaling through similar mechanisms. Calcineurin is a common target of cyclophilin-cyclosporin A and FKBP-FK506 complexes. Thus, we postulate that sirtinol also synergizes with CsA. Our future experiments will verify this hypothesis. 

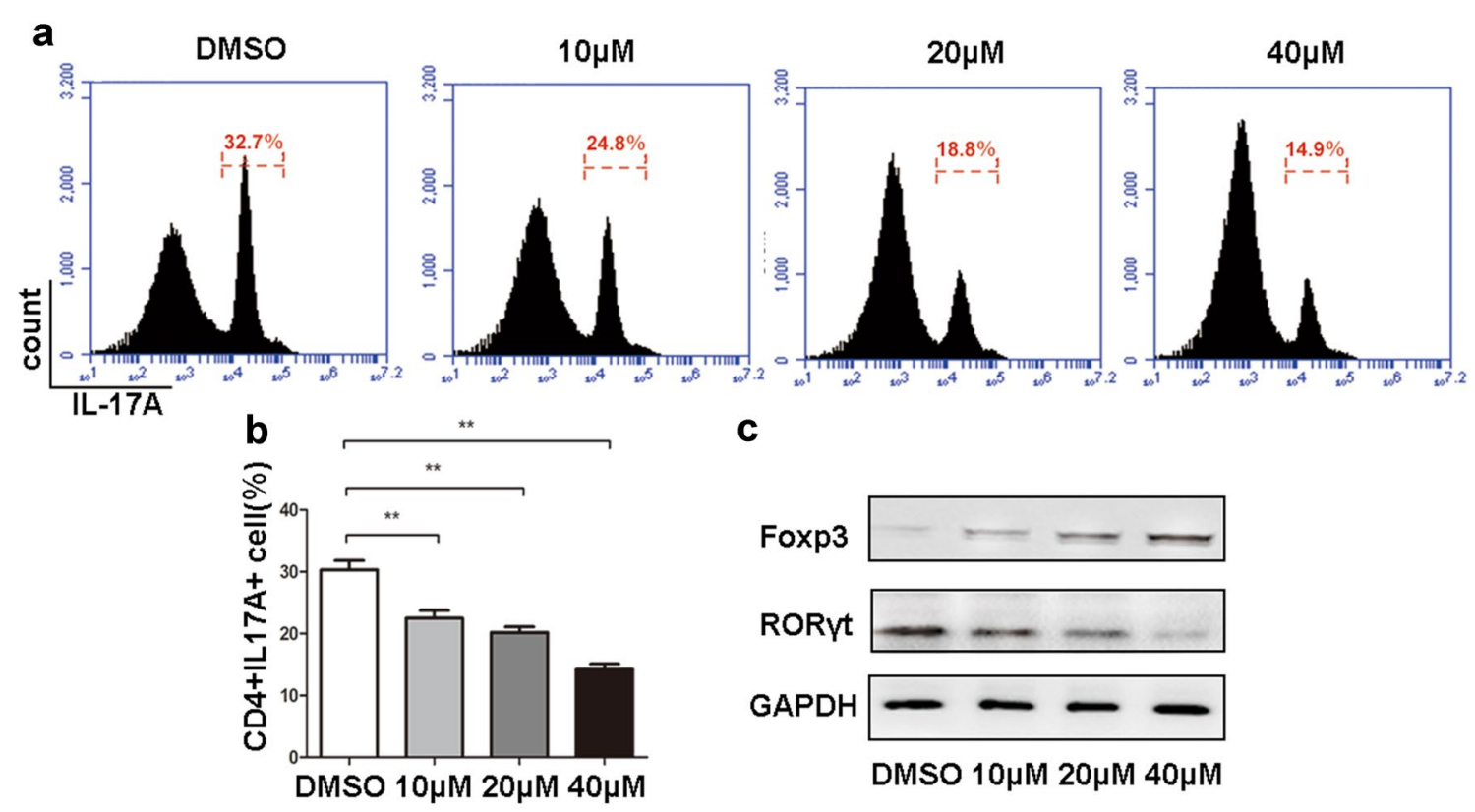

C
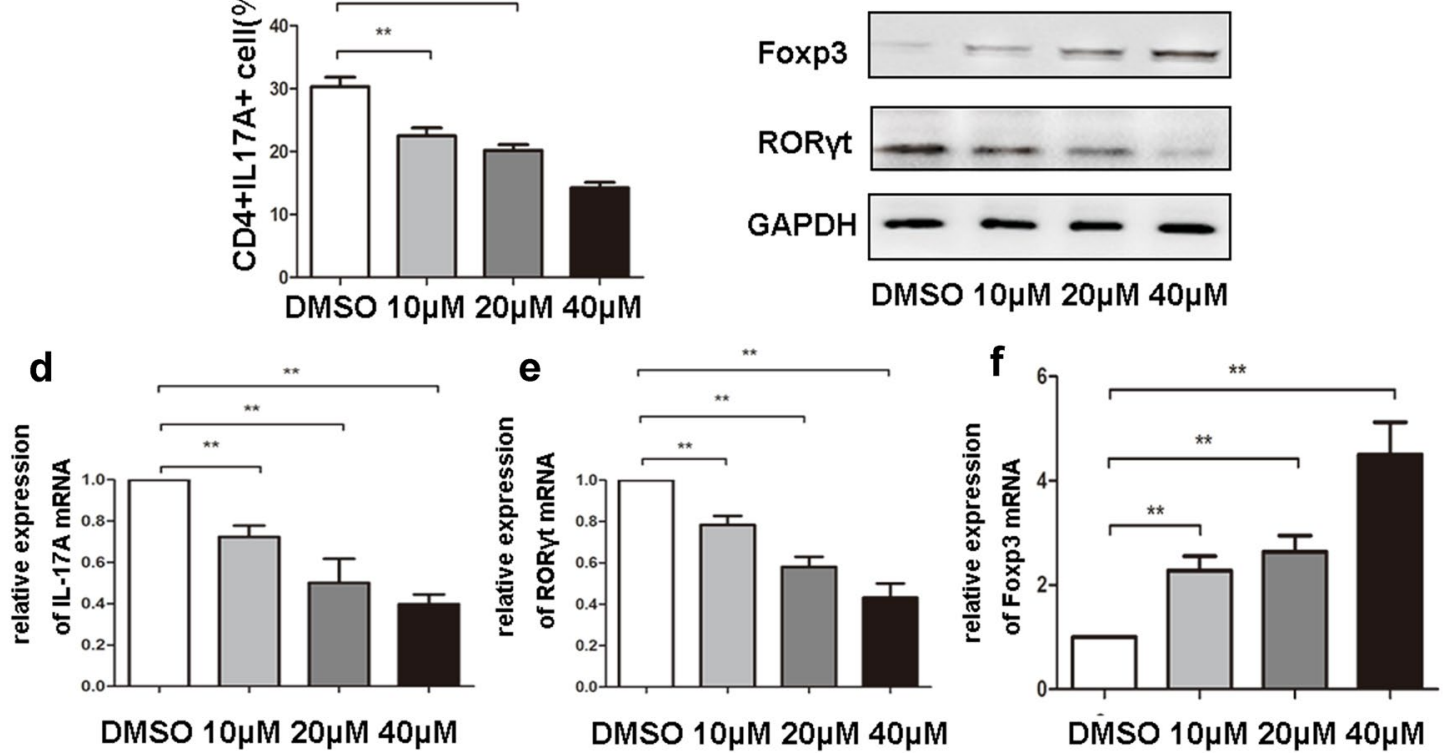

Fig. 4 Sirtinol regulates Th17/Treg balance. a Naive $C D 4^{+} \mathrm{T}$ cells were isolated from mouse spleen, and polarized for Th17 differentiation in the presence different concentration of sirtinol. IL-17A expressing CD4+ T cells were measured by flow cytometry. Shown are representative of five separate experiments. b Percentages of IL-17A+ cells were presented. cThe effect of sirtinol on the expressions of RORyt and Foxp3 measured by western blots. Shown are representative of five separate experiments. $\mathbf{d}-\mathbf{f}$ Gene expressions of IL-17A, RORyt, Foxp3 in CD4 ${ }^{+} \mathrm{T}$ cells measured by qPCR. The data are expressed as mean $\pm S D(n=5) . * P<0.05,{ }^{* * P}<0.01$, compared with DMSO group

In addition to its immunosuppressive effect, sirtinol also exerts other pharmacological functions. On one hand, sirtinol has been reported to suppress human cytomegalovirus (hCMV) infection and hCMV-induced activation of the molecular mechanisms of senescence and reactive oxygen species (ROS) production; [34] hCMV also causes serious infection in transplant recipients with reduced immune function [35]. On the other hand, pharmacological Sirt1 inhibition by sirtinol exerts a broad anti-tumor effect on several cancers, such as melanoma, breast cancer, and prostate cancer [19, 36-38]. Thus, sirtinol is not only an immunosuppressive agent that can be used to treat allograft rejection, but also a potential drug for preventing hCMV infections and the development of malignant tumors after organ transplantation. However, the side effects of sirtinol must also be considered. In our study, a high-dose sirtinol treatment did not prolong allograft survival and showed unexpected side effects. Therefore, additional studies are needed to evaluate the effects of sirtinol before clinical application.

\section{Conclusions}

Sirtinol prolongs allograft survival by regulating the Th17/Treg balance. Moreover, we identified synergistic effects of sirtinol and FK506 in prolonging allograft survival, and sirtinol synergizes with FK506 to promote Foxp3 expression. Therefore, sirtinol, a Sirt1 inhibitor, may be a promising immunosuppressive drug to prevent rejection reactions in combination with FK506. 


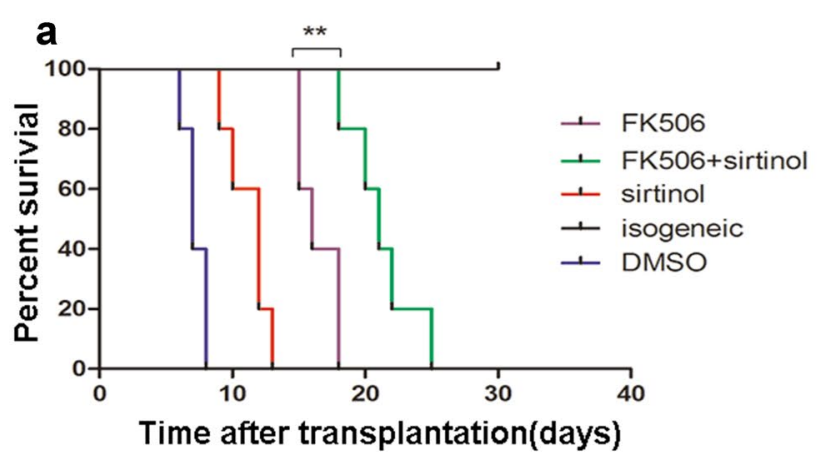

b

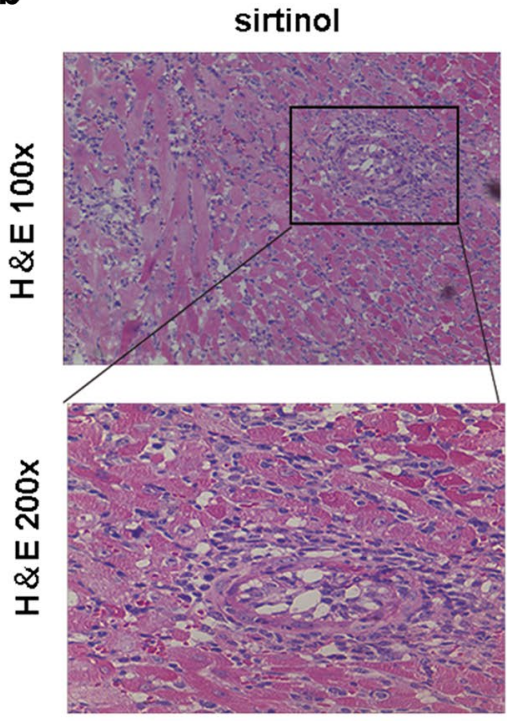

FK506
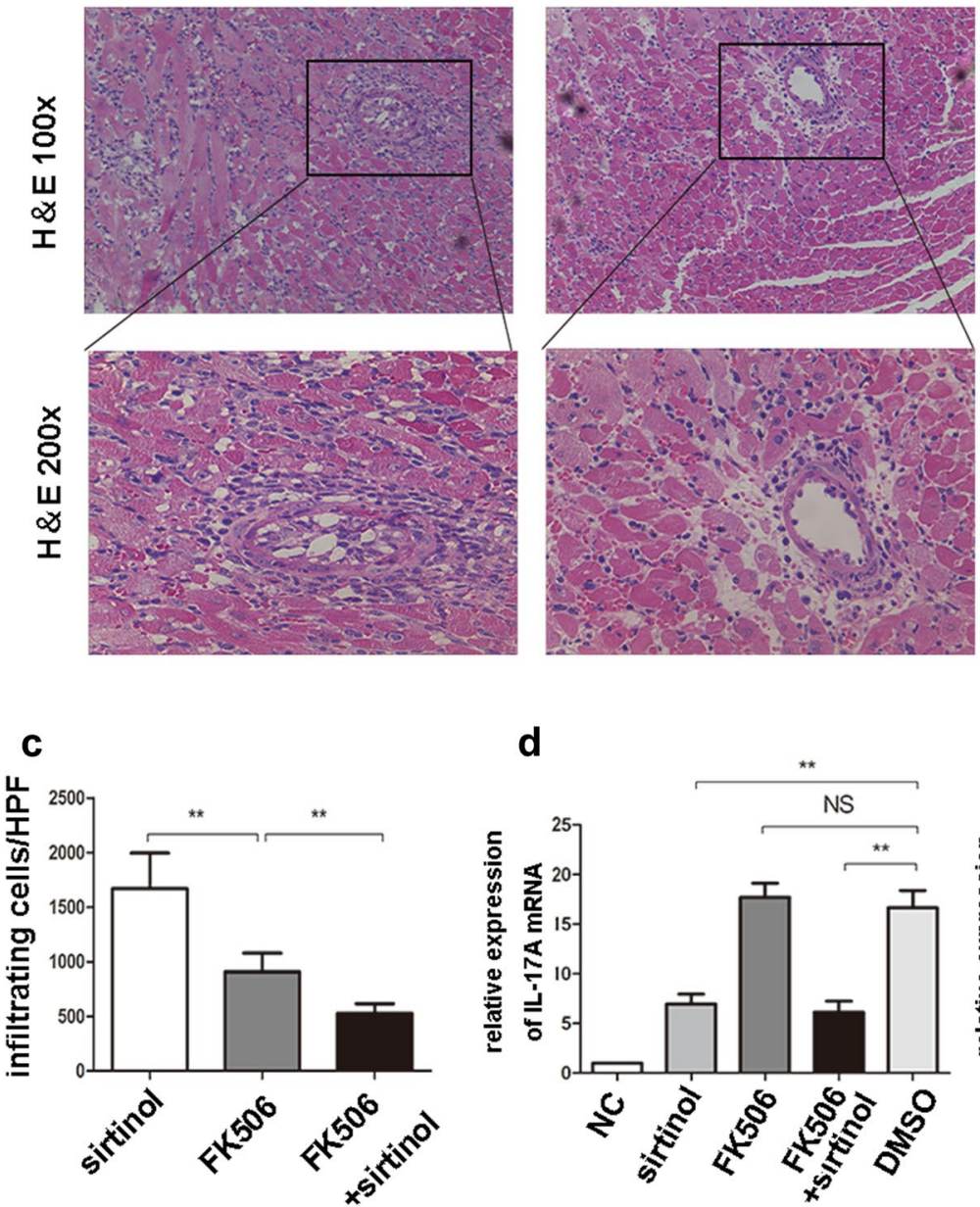

d

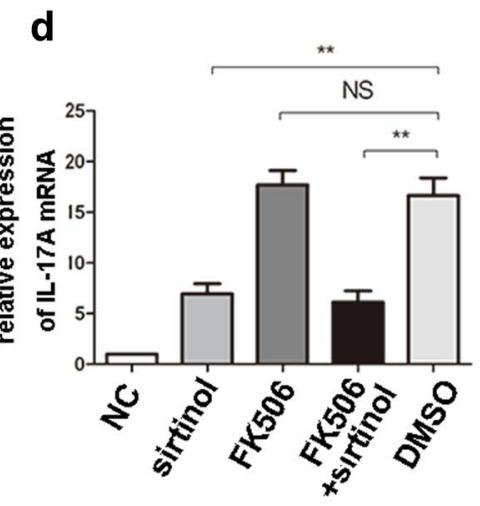

Fig. 5 Sirtinol synergizes with FK506 to prevent mouse cardiac allograft rejection. a Fully MHC-mismatched cardiac allograft recipients (BALB/C to C57BL/6) were treated with DMSO, sirtinol (1 mg/kg/day), FK506 (1 mg/kg/day) or sirtinol (1 mg/kg/day) —FK506 (1 mg/kg/day) combination by intraperitoneal injection since the day after transplantation until when cardiac arrest occurred. Graft survival was assessed every day. b Pathological examination of allografts harvested on day 12 post-transplantation. Shown are section graphs from the low power field and high power field. c Infiltrating cells per high power field were counted from five mice per group. $\mathbf{d}$, e IL-17A and Foxp3 mRNA were measured by qPCR. The data are expressed as mean $\pm S D(n=5)$. ${ }^{*} P<0.05,{ }^{* * P}<0.01$. NC negative control represented cardiac graft from isotransplantation 


\section{Abbreviations}

Sirt1: sirtuin 1; CNIs: calcineurin inhibitors; APC: antigen-presenting cell; HDAC: histone deacetylase; NAD: nicotinamide adenine dinucleotide; DMSO: dimethyl sulfoxide; ELISA: enzyme-linked immunosorbent assay; MHC: major histocompatibility complex; GVHD: graft-versus-host disease; FK506: tacrolimus.

\section{Authors' contributions}

Conceived, designed and supervised the project: QY, MZ and YW. Performed the experiments: QY, MZ, YW, SF, SH and QW. Analyzed data: QY, MZ and YW. Wrote the paper: QY, LW and QW. All authors read and approved the final manuscript.

\section{Author details \\ ${ }^{1}$ Institute of Organ Transplantation, Changzheng Hospital, Second Military Medical University, Shanghai, China. ${ }^{2}$ National Key Laboratory of Medical Immunology, Second Military Medical University, Shanghai, China. ${ }^{3}$ Depart- ment of Clinical Surgery, Chinese PLA General Hospital, 28th Fuxing Road, Beijing, China.}

\section{Acknowledgements}

We thank Meng Guo, Ph.D., for providing scientific suggestions on our work.

\section{Competing interests}

The authors declare that they have no competing interests.

\section{Consent for publication}

Not applicable.

\section{Data availability}

The data supporting the conclusions of this article is available to all interested researchers upon request.

\section{Ethical approval and consent to participate}

All animal experiments were conducted under the experimental protocol approved by the Ethics Review Committee for Animal Experimentation of the Second Military Medical University (Shanghai, China).

\section{Funding}

This work was supported by funding from the National Natural Science Foundation of China (81671577 and 81373171).

\section{Publisher's Note}

Springer Nature remains neutral with regard to jurisdictional claims in published maps and institutional affiliations.

Received: 3 May 2017 Accepted: 4 September 2017

Published online: 27 October 2017

\section{References}

1. Casey MJ, Meier-Kriesche HU. Calcineurin inhibitors in kidney transplantation: friend or foe. Curr Opin Nephrol Hypertens. 2011;20(6):610-5.

2. Ponticelli C, Scolari MP. Calcineurin inhibitors in renal transplantation still needed but in reduced doses: a review. Transpl Proc. 2010;42(6):2205-8.

3. Herrera $O B$, Golshayan D, Tibbott R, et al. A novel pathway of alloantigen presentation by dendritic cells. J Immunol. 2004;173(8):4828-37.

4. Oluwole OO, Depaz HA, Gopinathan R, et al. Indirect allorecognition in acquired thymic tolerance: induction of donor-specific permanent acceptance of rat islets by adoptive transfer of allopeptide-pulsed host myeloid and thymic dendritic cells. Diabetes. 2001;50(7):1546-52.

5. Zhou Y, Yang X, Zhang H, Jiang J. The roles of T helper type 17/regulatory T cells in acute rejection after liver transplantation in rats. Transplantation. 2015;99(6):1126-31.

6. Hanidziar D, Koulmanda M. Inflammation and the balance of Treg and Th17 cells in transplant rejection and tolerance. Curr Opin Organ Transpl. 2010;15(4):411-5.
7. Diller ML, Kudchadkar RR, Delman KA, Lawson DH, Ford ML. Balancing inflammation: the link between Th17 and regulatory T cells. Mediat Inflamm. 2016;2016:6309219.

8. Abadja F, Sarraj B, Ansari MJ. Significance of Thelper 17 immunity in transplantation. Curr Opin Organ Transpl. 2012;17(1):8-14.

9. Li Y, Shi Y, Huang Z, et al. CNI induced Th17/Treg imbalance and susceptibility to renal dysfunction in renal transplantation. Int Immunopharmacol. 2011;11(12):2033-8.

10. Finkel T, Deng CX, Mostoslavsky R. Recent progress in the biology and physiology of sirtuins. Nature. 2009;460(7255):587-91.

11. Gao B, Kong Q, Kemp K, Zhao YS, Fang D. Analysis of sirtuin 1 expression reveals a molecular explanation of IL-2-mediated reversal of T-cell tolerance. Proc Natl Acad Sci USA. 2012;109(3):899-904.

12. Kong S, McBurney MW, Fang D. Sirtuin 1 in immune regulation and autoimmunity. Immunol Cell Biol. 2012;90(1):6-13.

13. Zhang J, Lee SM, Shannon S, et al. The type III histone deacetylase Sirt1 is essential for maintenance of T cell tolerance in mice. J Clin Invest. 2009;119(10):3048-58.

14. Kwon HS, Lim HW, Wu J, Schnölzer M, Verdin E, Ott M. Three novel acetylation sites in the Foxp3 transcription factor regulate the suppressive activity of regulatory T cells. J Immunol. 2012;188(6):2712-21.

15. Beier UH, Wang L, Bhatti TR, et al. Sirtuin-1 targeting promotes Foxp3+ T-regulatory cell function and prolongs allograft survival. Mol Cell Biol. 2011;31(5):1022-9.

16. Lim HW, Kang SG, Ryu JK, et al. SIRT1 deacetylates RORgammat and enhances Th17 cell generation. J Exp Med. 2015;212(5):607-17.

17. Heltweg B, Gatbonton T, Schuler AD, et al. Antitumor activity of a smallmolecule inhibitor of human silent information regulator 2 enzymes. Cancer Res. 2006;66(8):4368-77.

18. Kozako T, Aikawa A, Shoji T, et al. High expression of the longevity gene product SIRT1 and apoptosis induction by sirtinol in adult T-cell leukemia cells. Int J Cancer. 2012;131(9):2044-55.

19. Wang J, Kim TH, Ahn MY, et al. Sirtinol, a class III HDAC inhibitor, induces apoptotic and autophagic cell death in MCF-7 human breast cancer cells. Int J Oncol. 2012;41(3):1101-9.

20. Kanda T, Sasaki R, Nakamoto S, et al. The sirtuin inhibitor sirtinol inhibits hepatitis A virus (HAV) replication by inhibiting HAV internal ribosomal entry site activity. Biochem Biophys Res Commun. 2015;466(3):567-71.

21. Liu FC, Day YJ, Liou JT, Lau YT, Yu HP. Sirtinol attenuates hepatic injury and pro-inflammatory cytokine production following trauma-hemorrhage in male Sprague-Dawley rats. Acta Anaesthesiol Scand. 2008;52(5):635-40.

22. Wang K, Zhang N, Li H. Improved technique of mouse heterotopic heart graft retransplantation. Microsurgery. 2006;26(3):200-2.

23. Zhang $X$, Han $S$, Kang $Y$, et al. SAHA, an HDAC inhibitor, synergizes with tacrolimus to prevent murine cardiac allograft rejection. Cell Mol Immunol. 2012;9(5):390-8.

24. Schramm A, Jasiewicz-Honkisz B, Osmenda G, et al. Th17 responses are not altered by natural exposure to seasonal allergens in pollen-sensitive patients. Allergy Asthma Clin Immunol. 2016;12:55.

25. Du C, Liu C, Kang J, et al. MicroRNA miR-326 regulates TH-17 differentiation and is associated with the pathogenesis of multiple sclerosis. Nat Immunol. 2009;10(12):1252-9.

26. de Mattos AM, Olyaei AJ, Bennett WM. Nephrotoxicity of immunosuppressive drugs: long-term consequences and challenges for the future. Am J Kidney Dis. 2000;35(2):333-46.

27. Carlson MJ, West ML, Coghill JM, Panoskaltsis-Mortari A, Blazar BR, Serody JS. In vitro-differentiated Th17 cells mediate lethal acute graft-versus-host disease with severe cutaneous and pulmonary pathologic manifestations. Blood. 2009;113(6):1365-74.

28. Yuan X, Paez-Cortez J, Schmitt-Knosalla I, et al. A novel role of CD4 Th17 cells in mediating cardiac allograft rejection and vasculopathy. J Exp Med. 2008;205(13):3133-44.

29. Itoh S, Nakae S, Axtell RC, et al. IL-17 contributes to the development of chronic rejection in a murine heart transplant model. J Clin Immunol. 2010;30(2):235-40.

30. Booth AJ, Csencsits-Smith K, Wood SC, Lu G, Lipson KE, Bishop DK. Connective tissue growth factor promotes fibrosis downstream of TGFbeta and IL-6 in chronic cardiac allograft rejection. Am J Transpl. 2010;10(2):220-30. 
31. Deteix C, Attuil-Audenis V, Duthey A, et al. Intragraft Th17 infiltrate promotes lymphoid neogenesis and hastens clinical chronic rejection. J Immunol. 2010;184(9):5344-51.

32. Graca L, Chen TC, Le MA, Cobbold SP, Howie D, Waldmann H. Dominant tolerance: activation thresholds for peripheral generation of regulatory $T$ cells. Trends Immunol. 2005;26(3):130-5.

33. Fallarino F, Grohmann U, Hwang KW, et al. Modulation of tryptophan catabolism by regulatory T cells. Nat Immunol. 2003;4(12):1206-12.

34. Mao G, Li H, Ding X, Meng X, Wang G, Leng SX. Suppressive effects of sirtinol on human cytomegalovirus (hCMV) infection and hCMV-induced activation of molecular mechanisms of senescence and production of reactive oxygen species. Mech Ageing Dev. 2016;158:62-9.
35. Snoeck R, Andrei G, Neyts J, et al. Inhibitory activity of S-adenosylhomocysteine hydrolase inhibitors against human cytomegalovirus replication. Antiviral Res. 1993;21(3):197-216.

36. Wilking MJ, Singh CK, Nihal M, Ndiaye MA, Ahmad N. Sirtuin deacetylases: a new target for melanoma management. Cell Cycle. 2014;13(18):2821-6.

37. Ota H, Tokunaga $E$, Chang K, et al. Sirt1 inhibitor, Sirtinol, induces senescence-like growth arrest with attenuated Ras-MAPK signaling in human cancer cells. Oncogene. 2006;25(2):176-85

38. Jung-Hynes B, Nihal M, Zhong W, Ahmad N. Role of sirtuin histone deacetylase SIRT1 in prostate cancer. A target for prostate cancer management via its inhibition. J Biol Chem. 2009;284(6):3823-32.

\section{Submit your next manuscript to BioMed Central and we will help you at every step:}

- We accept pre-submission inquiries

- Our selector tool helps you to find the most relevant journal

- We provide round the clock customer support

- Convenient online submission

- Thorough peer review

- Inclusion in PubMed and all major indexing services

- Maximum visibility for your research

Submit your manuscript at www.biomedcentral com/submit 